\title{
A Review of Conventional Fault Detection Techniques in Solar PV Systems and a Proposal of Long Range (LoRa) Wireless Sensor Network for Module Level Monitoring and Fault Diagnosis in Large Solar PV Farms
}

\author{
Aruoriwoghene Okere and M. Tariq Iqbal
}

\begin{abstract}
This paper reviews various faults that exist in large solar Photovoltaic (PV) systems. The faults are reviewed in their various classes based on the location and structure. Conventional solutions for fault detection and various research work in PV system monitoring and fault detection are reviewed. It is obvious that PV module level monitoring exhibit advantages over array or string monitoring. Therefore, the paper proposes the use of Long Range (LoRa) Wireless Sensor Networks (WSN) for PV module level monitoring and fault diagnosis. LoRa was proposed for this application due to the advantages it has over other wireless technologies which include long range of data transfer, low cost, low power consumption and multi sensor connection capabilities.
\end{abstract}

Index Terms - PV Module level faults, LoRa, Wireless Sensor Networks, Solar Energy, Renewable Energy.

\section{INTRODUCTION}

Energy demand is constantly on the rise due to increase in industrial activities, socio-economic activities, and lifestyles. This has made research into green energy a necessity. The primary aim of renewable energy technology research is conversion of renewable energy sources to electrical energy which can be fed to the utility grid or used locally. Solar energy no doubt is a very important source of renewable energy. However, since they are installed in outdoor environment, with exposure to harsh climatic and environmental conditions, the optimal performance of the solar system may be reduced. These conditions that reduce the performance of the solar system are known as faults. In [1], it was reported that an annual power loss due to fault is about $18.9 \%$. Therefore, to address these faults, a complete knowledge of meteorological data is essential, also constant monitoring of solar modules is crucial. Moreover, techniques for fault identification in real time need to be developed.

Photovoltaic module health monitoring is essential in reducing losses and improve efficiency of a PV plant system [2]. Various methods for detecting and identifying faults and failures have been studied. In [3], the authors mentioned that diagnosis method can be based on characteristics curve deviation analysis. This involves a comparison between the experimental measurements and model-based data of the PV system.

Published on December 30, 2020.

Aruoriwoghene Okere, Memorial University of Newfoundland, Canada. (corresponding e-mail: auokere@mun.ca)

M. Tariq Iqbal, Memorial University of Newfoundland, Canada.

\section{COMMON FAULTS AND FAILURES IN PV SYSTEMS}

Factors that reduce the output of a PV module are considered as faults. Faults may be temporary or permanent. Generally, some factors that are responsible for energy loss in PV systems include varying solar radiation level and operating temperatures, mismatch in PV module parameters, errors in maximum power point tracking, shading effects, aging, snow and dust accumulation on the PV modules and power converter losses [1,4]. Another study in Spain identifies the following faults in PV; yellowing and browning, delamination, bubbles in solar module, cracks in cells, defects in anti-reflective coating, hotspots caused usually when solar panels act as load, edge cell delamination, delamination over cells and interconnection, split encapsulation over cells and interconnections and protruding interconnections [5,6]. Faults in PV systems can be classified and defined based on the location and structure. For this review, common faults in the PV modules (DC side) will be enumerated [7].

\section{A. Arc Faults and Line to Line Faults}

Arc faults are due to discontinuity in current carrying conductors which results from solar disjoint, cell delamination, corrosion of connections or abrasion from other sources. This can also result from insulation breakdown from current carrying conductors. Line to line faults are due to accidental short circuit current between two points in a string with different voltage potentials.

\section{B. Degradation Faults}

This is due to delamination, cracks in cells, yellowing and browning, bubbles in solar module, defects in anti-reflective coating and delamination over cells and interconnections leading to degradation and increase in internal resistance.

\section{By-pass Diode Faults and Bridging Faults}

By-pass diode faults can be due to incorrect connection that results in short circuits. While bridging faults are due to low resistance connection between two points of different potential in string of Modules or in cabling.

\section{Open Circuit Faults and Mismatch Faults}

Open circuit faults occur as a result of one or more of the following reasons: physical breakdown of panel to panel cables or joints, objects falling on PV panels, loose termination of cables, inappropriate handling of connectors at junction boxes. Mismatch faults are usually due to partial shading, soiling, snow, or dirt covering and hot spots. 


\section{REVIEW OF EXISTING PV System FAUlt DETECTION TECHNIQUES}

Efforts are been made in recent times to understand the different classification of faults with the aim of developing techniques to detect and locate the type of fault present in a PV system to improve the reliability and lifetime of the system. This review is focused on fault detection on the PV modules; therefore, some techniques have been documented from [1].

\section{A. Independent Meteorological Data Collection Technique}

This technique uses external devices such as LCR (Inductance, capacitance or resistance) meters and signal generators independent of climate data for fault detection. A technique that applies the earth capacitance measurement to detect discontinuities of PV module in a string was proposed in [8]. In this technique, the time domain reflectometry (TDR), which applies the voltage signal into the string and observes the signal response waveforms, was applied to the PV strings containing faults to detect the fault and locate its position. With this technique, faults such as degradation and disconnection between strings of modules were detected.

\section{B. Measurement of Current-Voltage (I-V) Characteristics}

Faults are detected through the measurement of I-V characteristics at the output terminals [9]. Various researchers have used this technique for faults detection. In [10], a real time microcontroller-based system is used to monitor, supervise, and control the PV system. Additionally, the developed system is easily connected to a network, and thus it allows access to information about PV systems installed over a wide area. Also, a similar system was proposed in [11] that detected the location of a fault in grid connected PV systems automatically through voltage and current indicators by monitoring the ratio between $\mathrm{AC}$ and DC power. The software tool developed can detect faults on both the AC and DC side of the PV plant. Faults that can be detected using this method include fault in a photovoltaic string, fault in an inverter, and a general fault that may include partial shading, PV ageing, or maximum power point tracking (MPPT) error.

\section{Comparing the Actual Output and Simulated Output of the PV system}

In this technique, a comparison between the simulated PV output from modelling and the actual measured output is made. The difference is used as an indicator for fault detection. The expected output power is determined from PV models along with other variable by establishing a threshold within the normal operations of the PV system. If the measured output exceeds the threshold, then the system is considered to be faulty.

\section{Analyzing the power losses}

Fault detection is based on analysis of power losses in the PV system by calculating the difference in the measured or monitored power and the simulated results. This technique was proposed in [12] for supervision and fault detection in PV systems. With the technique developed, faults on the DC side of the PV system can be identified. The system defines two power losses indicators as thermal capture losses and miscellaneous capture losses. The processing of these indicators allows the supervisory system to generate a faulty signal as indicator of fault detection in the PV system operation.

\section{E. The Use of Artificial Neural Networks}

In this fault detection technique, the system model is trained to learn different conditions that corresponds with a particular data type. Introductions of new conditions are identified based on previous training and can be used for fault detection. Since PV system output can vary under different climatic conditions, it can become difficult to define theoretical boundaries for fault detection. However, training a model for certain input-output detection can overcome this limitation. A trained system has been used to identify faults in PV array due to partial shading and inverter losses [13]. This technique is used to provide a simplified maintenance structure for PV systems. This technology employs diagnostic criteria databases to analyze data acquired from the PV systems and this database are updated and verified regularly. Also, a maintenance advice is provided after adequate diagnosis has been carried out. This technology is highly useful, because it greatly simplifies the servicing and maintenance of PV systems. Also, artificial neural network was used in [14] for classification of different faults in PV arrays. The model of the Artificial Neural Networks (ANN) is developed in such a way that it inputs parameters such as voltage and current at maximum power point and temperature of the PV and then outputs the type of fault present in the system.

\section{F. Infrared or Thermal Imaging}

This is a popular method for fault detection in PV systems. Several types of faults such as poor conductors, short circuits and joule heating effects leads to localized heat generation and this is what thermal imaging is based on. In series connected PV cells, some cells generate less energy than others when faulty, as a result becomes reversed biased and acts as load and hence dissipate heat. This results in the formation of temperature gradient that appears as a bright spot during thermal imaging. Thermal imaging can be carried out in two ways.

1) Forward bias imaging: In forward bias imaging, the module is in the forward bias condition and connected to the power supply. The current (which is two times greater than the module short circuit current) will flow through the module which leads to heating of the modules. The IR images are then recorded using the IR camera and image processing is carried out to identify the type and location of the fault. This method can be used to identify faults such as hotspot, loose connection and increase in series resistance.

2) Reverse bias imaging: In reversed bias imaging, the same procedure as in forward bias imaging is carried out but with the module connected to the power supply in a reverse biased condition. Faults such as ohmic shunts are identified using this method. 


\section{PV Module LeVEl Monitoring AND FAUlt DETECTION}

It is important to point out that most of the fault detection techniques that have been reviewed in the preceding sections are applied on the PV systems itself without any information about the converters or on the $\mathrm{AC}$ side. The importance of fault detection on the PV modules are outlined in the following section.

The Advantages of PV module level monitoring and fault detection include:

1) Effective localization of faults within a large PV system. Hence, reducing maintenance cost as faults can be identified at the specific PV module.

2) Minimize the impact of failure to reduce downtime in large arrays in order to improve energy production.

3) Data presentation and full visibility of technical performance at the module level for accurate forecasting of overall performance of the PV system.

4) Remote troubleshooting and access to real time system data to aid root cause fault analysis [15].

\section{REVIEW OF PREVIOUS WORK DONE IN MONITORING AND FAULT DETECTION ON PV SYSTEMS USING WIRELESS SENSOR NETWORKS}

Research on PV system module monitoring and fault detection have been done in a vast scale and are still ongoing. Some of which involve sensing and monitoring of electrical parameters and climatic variables on the PV array instantaneously or overtime, communicating these data between sensors and the external gateway where data is analyzed. Therefore, sensors or nodes together with a gateway communicating over a wireless network is referred to a Wireless Sensor Networks (WSN).

An intelligent fault detection method of PV array was proposed using Zigbee wireless sensor Networks for fault diagnosis [16]. An acquisition circuit for measuring the different characteristics value was developed. Also, a PIC microcontroller and ZigBee module was used to construct a fault meter based on neural networks to access the PV power generation system to a high degree of accuracy. However, fault detection was not carried out on PV module level and the WSN only covers short distance $(100 \mathrm{~m})$.

An internet-based monitoring and protection of PV smart grid system was designed by Sholehi and his team [17]. In this design, a voltage and current sensor tool was used to monitor voltage and current data which is then converted to electrical power. The system is also capable of carrying out power protection to load to ensure that power supplied does not exceed the power specification of the load by using a relay for switching. The monitoring and protection system makes use of wireless communication through the internet with a Wi-Fi module. However, monitoring is done on the array level, which is not time and cost effective.

In [18], an internet of things (IoT) based solar panel fault monitoring and control system was proposed. Data acquisition sensors are essentially used to obtain current, voltage, temperature, light, and dust data. Zigbee is used as the wireless communication mechanism and hence only short-range communication can be covered.
A method of PV monitoring of PV module from a string is carried out in [19]. Here, the open circuit voltage and current, short circuit voltage and current of the panels connected to a string are monitored independent of their operation. The PV panel is separated from the string and measurement in the open circuit and short circuit condition are taken out. These parameters are sent to the coordinator node via wireless communication for analysis and are also stored in the data memory of the microcontroller unit. In this way, the reliability of the PV system is monitored with the help of a wireless sensor network and therefore leads to localization of fault in the case of malfunctioning of PV panel in a string.

A system that automatically detects fault using IoT is proposed by authors in [20]. Current sensors are connected to the panel to obtain current data. These data are sent to the monitoring center through a Wi-Fi module. At the monitoring center, the data is analyzed, and faults detected if any.

A monitoring system for online fault detection and classification of PV Plants was designed in [21]. A monitoring system that measures electrical and environmental data instantaneously and historically is proposed to estimate the efficiency of the PV plant.

Considering a large PV system with a high number of solar PV modules, to achieve module level monitoring may seem a cumbersome and expensive task. This is due to the fact that effective PV module level monitoring for performance and health analysis involves real-time observations of the various modules of the large system and possible remote analysis conducted on the obtained results. This process requires carrying out the above stated procedure for each module in the system for effective localization of faults and possible resolution. The incorporation of the Internet of Things (IoT) and the employment of low power, low cost communication and wireless sensor networks are a cost effective and feasible approach to achieve PV module level monitoring in a large PV system. Wireless communication has numerous benefits over wired communication.

Therefore, the benefits of the wireless sensor networks include cost benefit analysis, continuous parameter monitoring, access and evaluation of output from individual modules, reliable and secure means of communication and data transfer, scalability and hence can accommodate any new nodes or devices at any time, flexibility and Accessibility since all WSN nodes can be accessed through centralized monitoring system.

A comparison of all low power wide-area network (LPWAN) that can be used for PV module monitoring and fault detection are shown in Table I.

From the publications reviewed in this work, to the best of the authors' knowledge, no work has applied a LoRa Wireless Sensor Network for PV module level fault monitoring and Diagnosis. This paper proposes to apply the LoRa WSN for this purpose. In this paper, only the architecture and advantages of employing LoRa will be discussed. The full design and development of the system will be focused on in future works as this will not be covered in this paper. 
TABLE I: COMPARISON OF LPWAN NETWORKS

\begin{tabular}{lccccc}
\hline \hline Specification & LoRa & ZigBee & Sigfox & Bluetooth & $\begin{array}{c}\text { Dash } \\
7\end{array}$ \\
\hline $\begin{array}{l}\text { Low Cost of System } \\
\text { Components }\end{array}$ & $\mathrm{X}$ & $\mathrm{X}$ & & $\mathrm{X}$ & \\
$\begin{array}{l}\text { High Interference } \\
\text { Immunity }\end{array}$ & $\mathrm{X}$ & & & & \\
$\begin{array}{l}\text { Long Distance } \\
\text { Coverage }\end{array}$ & $\mathrm{X}$ & & & & \\
$\begin{array}{l}\text { Low power } \\
\text { Consumption }\end{array}$ & $\mathrm{X}$ & $\mathrm{X}$ & & $\mathrm{X}$ & \\
$\begin{array}{l}\text { Multipoint } \\
\text { Connection }\end{array}$ & $\mathrm{X}$ & $\mathrm{X}$ & $\mathrm{X}$ & & $\mathrm{X}$ \\
$\begin{array}{l}\text { High Security } \\
\text { Sensor Direct }\end{array}$ & $\mathrm{X}$ & $\mathrm{X}$ & $\mathrm{X}$ & & \\
$\begin{array}{l}\text { Connectivity } \\
\text { Expansion }\end{array}$ & $\mathrm{X}$ & $\mathrm{X}$ & & $\mathrm{X}$ & \\
Capability & $\mathrm{X}$ & $\mathrm{X}$ & & $\mathrm{X}$ & \\
\hline \hline
\end{tabular}

\section{PRoposing LoRA WIRELESS SENSOR NETWORK For} PV MOdUle LEVEl MONITORING AND FAULT DETECTION

LoRa is a long-range wireless communication system promoted by LoRa alliance. LoRa has received lots of attention due to their ability to offer a reliable means of communication for extensive monitoring and management applications such as health monitoring, smart metering, and environment monitoring [22].

Lora has an adjustable data rate and a higher level of coverage than the other low power wide area networks (LPWAN) standards thus making it a promising technology for applications in wireless sensor networks. LoRa technology used wide band frequency modulated pulses called Chirps for encoding to achieve a spreading gain as such provides successful reception of packets at low SNR regimes [22]. There are two layers in LoRa; Physical layer using the chirp spread spectrum (CSS) modulation technique developed by Semtech and allows for low power, long range and low throughput communication. It operates on the 433 $\mathrm{MHz}, 868 \mathrm{MHz}$ and $915 \mathrm{MHz}$ frequencies depending on the deployment area/region. Data rates can get up to $50 \mathrm{Kbps}$ when channel aggregation is employed, and payload size can be between 2-255 octets [23]. The second layer is MAC layer Protocol for multiple access architecture which is provided by LoRaWAN. This enables many end devices to communicate with a gateway using LoRa Modulation.

\section{A. LoRaWAN Architecture}

A typical architecture of LoRa is a star of star topology as shown in Fig. 1 and it includes different types of devices. The end devices communicate with the gateway using LoRaWAN. The gateway acts as bidirectional relay and typically forwards the LoRaWAN frames to the network server over a backhaul interface with a higher throughput for example $3 \mathrm{G}$ or Ethernet. The network server is responsible for decoding the packets sent by the devices and generating the downlink packet which will be transmitted back to the devices [23].

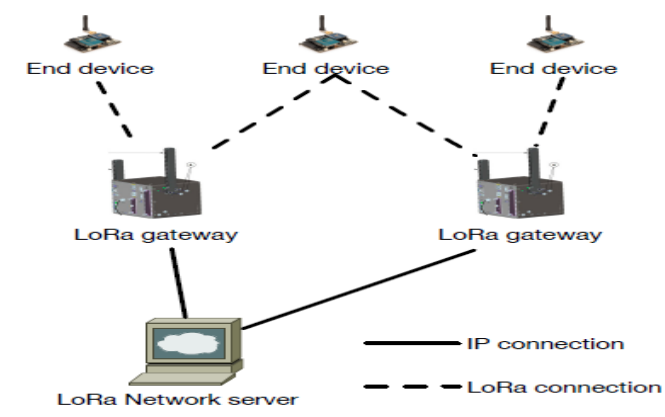

Fig 1. LoRaWAN Architecture [23].

\section{B. Physical layer of LoRaWAN}

LoRa employs chirp spread spectrum modulation which encode information by using frequency chirps where the frequency varies linearly overtime. This modulation is unaffected by Doppler effects. As a result of this linearity, frequency offsets between the transmitter and receiver are equivalent to timing offsets. This offset can usually be eliminated in the decoder [22]. The frequency offset between transmitter and receiver can reach up to $20 \%$ of bandwidth without impacting decoding performance as such low cost LoRa transmitter. However, LoRa transmitters are required to lock on the received frequency chirps therefore sensitivity of the order of $-130 \mathrm{dBm}$ is offered [23].

The symbol duration of LoRa is longer than that of a typical amplitude modulated (AM) signal generated by frequency hopping spread spectrum system, therefore errors due to interference are easily corrected using forward error correction codes (FEC).

The typical out of channel sensitivity and co-channel rejection of LoRa receivers is typically $90 \mathrm{~dB}$ and $20 \mathrm{~dB}$ outperforming traditional modulation schemes such frequency shift keying (FSK) and as such LoRa is well suited for low power and long-range transmission [24].

Bandwidth (BW), spreading factor (SF) and code rate (CR) are parameters for LoRa modulation. These parameters influence the effective bitrate of the modulation, its resistance to noise and its ease of decoding [23].

A LoRa symbol is composed of 2SF chirps which covers the entire frequency band. The chirp rate depends only on the bandwidth since these are 2SF chirps in a symbol, a symbol can effectively encode SF bits of information. The symbol duration (Ts), bandwidth (BW) and spreading factor (SF) are related by equation (1).

Bandwidth (BW) and spreading factor (SF) are related by equation (1).

$$
T_{S}=\frac{2^{S F}}{B W}
$$

where,

$\mathrm{BW}=$ Bandwidth, $\mathrm{SF}=$ Spreading Factor .

Also, FEC codes are included in LoRa.

The Code rate $C R=\frac{4}{(4+n)}$ where $\mathrm{n} \in\{1$ 1.2.3.4 $\}$.

Therefore, the useful bit rate $\mathrm{R}_{\mathrm{b}}$ is given in equation (2).

$$
R_{b}=S F \times \frac{B W}{2^{S F}} \times C R
$$


It is important to note that these parameters also influence the decoder sensitivity. Generally, an increase in bandwidth reduces the receiver sensitivity. However, receiver sensitivity is increased with an increase in spreading factor. Also, increasing the code rate reduces the packet error rate. In depth explanations of LoRa communication system has been carried out in [22] [23] [24] and [25].

\section{Advantages of LoRaWAN in PV module Level Monitoring}

LoRa WSN was reviewed in this paper to be employed for PV module level monitoring and fault diagnosis for some advantages surpassing its contemporaries such as the Zigbee, Bluetooth, Sigfox and DASH. These advantages include:

1) Long Range Communication: From a range test carried out by [25], it was observed that LoRa achieved a communication range of $4 \mathrm{~km}$ in an urban area with various forms of obstructions. Therefore, it can be deduced that in areas with less obstructions such as buildings, LoRa will achieve a much higher range for data transfer especially in large PV farms.

2) Low Cost: With source codes for programming the various components of the LoRa WSN obtained from an open source code site GITHUB, and the various components that will be employed such as the Arduino and the Raspberry Pi being very low cost materials. LoRa also operates on the Industrial, Scientific, and Medical frequency band of each region, hence there are no additional cost of using LoRa for sensor data transfer. Therefore, an additional elaborate cost will be not be incurred in the installation of PV farms with fault detection capabilities.

3) Low Power Consumption: LoRa communication consumes as low as $100 \mathrm{~mW}$ of power for data transfer which is a low value compared to the other communication technologies that have been used in the past.

4) Multi-sensor Connection Capabilities: LoRa communication technology permits the connection of a high number of sensors, which is a highly desirable feature for the WSN applications such as PV module level monitoring [28]. With many sensors supported, more data for monitoring and diagnostics can be acquired simultaneously.

\section{CONCLUSION}

In this paper, various faults and the fault detection methods that are existent in PV were reviewed. PV module level monitoring which is a very important application to achieve effective performance of the PV generation system through effective localization of faults in a solar PV system was also reviewed and its advantages enumerated. Finally, LoRa Wireless Sensor Network was proposed as an effective solution for the development of a low-cost PV module level monitoring and fault diagnosis. The advantages for which LoRa was chosen for this application were also explained in the work. In the design, we plan to sense, voltage, current, temperature and solar irradiance level of each PV module in a string of a large solar system.
A self-powered ultra-low power microcontroller with a LoRa module will measure those PV module parameters and transmit to a LoRa gateway. One LoRa gateway will receive signals from all PV modules and store that for local analysis and presentation. The designed system may or may not be connected to the internet. Collected data will be analyzed using an algorithm running on a low power single board computer to identify module faults. Alerts will be sent if the system detected any fault in PV modules. Detailed design and test result of the proposed method will be presented in the future publications.

\section{ACKNOWLEDGMENT}

We wish to acknowledge the Niger Delta Development Commission of the Federal Republic of Nigeria for the funding the carry out this research. Also, we acknowledge the Faculty of Engineering and Applied Sciences, Memorial University of Newfoundland for providing an enabling environment for research work.

\section{REFERENCES}

[1] S. Madeti and S. Singh, "A comprehensive study on different types of faults and detection techniques for solar photovoltaic system", Solar Energy, vol. 158, pp. 161-185, June 2017.

[2] V. Kongphet, A. Migan-Dubois, C. Delpha, D. Diallo and J. Lechenadec, "Photovoltaic Fault Detection and Diagnosis: Which Level of Granularity for PV Modeling?," Presented at 2020 Prognostics and Health Management Conference (PHM-Besançon), Besancon, France, May 4-7.

[3] M. Köntges, S. Kurtz, C. Packard, U. Jahn, K. Berger, K. Kato Kazuhilo, T. Friesen, H. Liu, I. Van, J. Wohlgemuth, D. Miller, M. Kempe, P. Hacke, F. Reil, N. Bogdanski, W. Herrmann, C. Buerhop, G. Razongles, and G. Friesen,". Review of Failures of Photovoltaic Modules" January 2014.

[4] S. Madeti and S. Singh, "Online modular level fault detection algorithm for grid-tied and off-grid PV systems", Solar Energy, vol. 157, pp. 349-364, November 2017

[5] M. Munoz, M. Alonso-García, N. Vela and F. Chenlo, "Early degradation of silicon PV modules and guaranty conditions", Solar Energy, vol. 85, no. 9, pp. 2264-2274, September 2011.

[6] A. Houssein, N. Heraud, I. Souleiman and G. Pellet, "Monitoring and fault diagnosis of photovoltaic panels", Presented at the 2010 IEEE International Energy Conference, Manama, Bahrain, December 18 22, 2010.

[7] M. Sabbaghpur Arani and M. Hejazi, "The Comprehensive Study of Electrical Faults in PV Arrays", Journal of Electrical and Computer Engineering, vol. 2016, pp. 1-10, December 2016.

[8] T. Takashima, J. Yamaguchi and M. Ishida, "Fault detection by signal response in PV module strings," Presented at the 33rd IEEE Photovoltaic Specialists Conference, San Diego, CA, USA, May $11-$ 162008.

[9] P. Lin, Y. Lin, Z. Chen, L. Wu, L. Chen and S. Cheng, "A Density Peak-Based Clustering Approach for Fault Diagnosis of Photovoltaic Arrays", International Journal of Photoenergy, vol. 2017, pp. 1-14, March 2017.

[10] M. Benghanem and A. Maafi, "Data acquisition system for photovoltaic systems performance monitoring," IEEE Transactions on Instrumentation and Measurement, vol. 47, no. 1, pp. 30-33, Feb. 1998

[11] W. Chine, A. Mellit, A. Pavan and S. Kalogirou, "Fault detection method for grid-connected photovoltaic plants", Renewable Energy, vol. 66, pp. 99-110, June 2014 .

[12] A. Chouder and s. Silvestre, "Automatic supervision and fault detection of PV systems based on power losses analysis", Energy Conversion and Management, vol. 51, no. 11, pp. 1929-1937, October 2010.

[13] Y. Yagi, H. Kishi, R. Hagihara, T. Tanaka, S. Kozuma, T. Ishida, M. Waki, M. Tanaka and S. Kiyama, "Diagnostic technology and an expert system for photovoltaic systems using the learning 
method", Solar Energy Materials and Solar Cells, vol. 75, no. 3-4, pp. 655-663, 2003

[14] Z. Li, Y. Wang, D. Zhou and C. Wu, "An Intelligent Method for Fault Diagnosis in Photovoltaic Array", Communications in Computer and Information Science, pp. 10-16, 2012.

[15] "Monitoring Solar Panel Efficiency. Available: https://www.solaredge.com/products/pv-monitoring/ [Accessed: 30August- 2020].

[16] K. Chao, P. Chen, M. Wang and C. Chen, "An Intelligent Fault Detection Method of a Photovoltaic Module Array Using Wireless Sensor Networks", International Journal of Distributed Sensor Networks, vol. 10, no. 5, p. 540147, May 15, 2014

S. H. Pramono, S. N. Sari and E. Maulana, "Internet-based monitoring and protection on PV smart grid system," Presented at the 2017 International Conference on Sustainable Information Engineering and Technology (SIET), Malang, November 24 - 252017.

[17] S. Karthik, M. Mahalakshmi, R. Mahitha and S. Meena, "IoT Based Solar Panel Fault Monitoring and Control", International Journal of Information and Computing Science, vol. 6, no. 3, pp. 238-243, March 2019.

[18] S. Ayesh, P, Ramesh and S. Ramakrishnan, Design of wireless sensor network for monitoring the performance of photovoltaic panel. 1-6. October 2017

[19] M. Hariprabhu and K. Sundararaju, "IoT based Fault detection in Solar Panel using Arduino UNO with Wi-Fi Module ESP 8266", International Journal of Recent Technology and Engineering (IJRTE), vol. 8, no. 4, pp. 2630-2633, November 2019.

[20] A. Lazzaretti, C. Costa, M. Rodrigues, G. Yamada, G. Lexinoski, G. Moritz, E. Oroski, R. Goes, R. Linhares, P. Stadzisz, J. Omori and R. Santos, "A Monitoring System for Online Fault Detection and Classification in Photovoltaic Plants", Sensors, vol. 20, no. 17, p. 4688, August 2020.

[21] T. Ameloot, P. Van Torre and H. Rogier, "A Compact Low-Power LoRa IoT Sensor Node with Extended Dynamic Range for Channel Measurements", Sensors, vol. 18, no. 7, p. 2137, July 2018.

[22] A. Augustin, J. Yi, T. Clausen and W. Townsley, "A Study of LoRa: Long Range \& Low Power Networks for the Internet of Things", Sensors, vol. 16, no. 9, p. 1466, September 2016.

[23] A. Springer, W. Gugler, M. Huemer, L. Reindl, C. C. W. Ruppel and R. Weigel, "Spread spectrum communications using chirp signals," Presented at IEEE/AFCEA EUROCOMM 2000. Information System for Enhanced Public Safety and Security, Munich, Germany, 2000.

[24] C. Ndukwe, M. Tariq Iqbal, X. Liang, J. Khan and L. Aghenta, "LoRa-based communication system for data transfer in microgrids" AIMS Electronics and Electrical Engineering, vol. 4, no. 3, pp. 303325, September 2020.

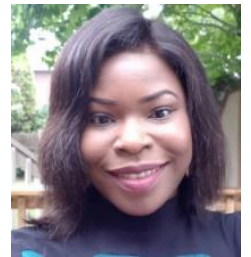

Aruoriwoghene Okere received her B.Eng Degree in Computer Engineering from University of Benin, Benin City, Nigeria in 2011. She became a Graduate Assistant at the same University and Department after her compulsory national Youth Service in 2013. She is presently serving as a Lecturer II. She received her MSc. Degree in Electronic and Electrical Engineering from the University of Leeds UK in 2015. She is currently pursuing her PhD in Electrical Engineering at the Memorial University of Newfoundland, Canada. Her research interest is in the area of Communication Application to Power Systems and renewable energy systems.

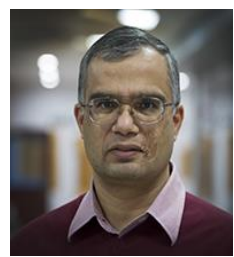

M. Tariq Iqbal received his B.Sc. (EE) at University of Engineering and Technology, Lahore in 1986, his M.Sc. Nuclear Engineering at CNS Quaid-e-Azam University, Islamabad in 1988 and $\mathrm{Ph} . D$. in Electrical Engineering at Imperial College London in 1994. From 1988 to 1991 and again from 1995 to 1999 , He worked at Pakistan Institute of Engineering and Applied Sciences (PIEAS), Islamabad. From 1999 to 2000, he also worked as an Associate Professor at Riphah International University. Since 2001, He has been working at Faculty of Engineering and Applied Science, Memorial University of Newfoundland. He teaches many electrical engineering courses e.g. electrical engineering design, process control and instrumentation, renewable energy systems, filters synthesis. Currently, his research focuses on modeling and control of hybrid renewable energy systems and with strong interest in consumer electronics and instrumentation. 